\title{
Social perceptions of warmth and competence influence behavioral intentions and neural processing
}

\author{
Jeremy C. Simon ${ }^{1}$ (D) Nadya Styczynski ${ }^{1}$ - Jennifer N. Gutsell ${ }^{1}$ \\ Published online: 21 January 2020 \\ (C) The Psychonomic Society, Inc. 2020
}

\begin{abstract}
Perceptions of the primary social dimensions, warmth and competence, determine how we view and relate to social targets. To discern how warmth and competence might affect neural processing and its downstream behavioral consequences, we manipulated impressions of targets' warmth and competence and then measured intentions toward the target and motor resonance, a neural process previously linked to social processing. While EEG was recorded, 66 participants watched videos of people performing a simple motor activity and completed a measure of hypothetical intentions to help or harm. Both perceptions of warmth and competence predicted an increase in helping intentions. Moreover, participants showed the least motor resonance with high competence-medium warmth targets, suggesting the importance of both social dimensions in driving neural simulation of targets' actions. Perceptions of a person's warmth and competence can affect not only how others might intend to treat them, but also how they might process their basic experiences on a neural level.
\end{abstract}

Keywords Social perception $\cdot$ Motor resonance $\cdot$ Mu-suppression $\cdot$ Warmth $\cdot$ Competence

\section{Introduction}

As perception informs action, social perception informs social interaction (Knoblich \& Sebanz, 2006). Social perception falls along two primary dimensions: warmth and competence (Abele \& Wojciszke, 2007; Asch, 1946; Fiske, Cuddy, Glick, \& Xu, 2002; Judd, James-Hawkins, \& Yzerbyt, 2005; for review, Abele \& Wojciszke, 2014), which distinguish intention and capability. Impressions of a social target along these dimensions influence the observer's reaction to and interaction with that person (Abele \& Bruckmüller, 2011). To investigate how perceptions of warmth and competence may affect neural action processing and downstream behavioral effects, we examined neural resonance - the similar activation of an observer's neural system during an action and during

Jeremy C. Simon and Nadya Styczynski contributed equally to this work.

Electronic supplementary material The online version of this article (https://doi.org/10.3758/s13415-019-00767-3) contains supplementary material, which is available to authorized users.

Jeremy C. Simon

jsimon13@brandeis.edu

1 Psychology Department, Brandeis University, MS062, 415 South Street, Waltham, MA 02453, USA mere observation of the same action - and relate it to intentions toward targets varying in perceived warmth and competence. We show that perceptions of targets' warmth and competence influence how perceivers neurally simulate their actions within their own sensorimotor system and the likelihood of the perceiver intending to help or harm the targets.

\section{Dimensions of social perception}

Competence is the ability to achieve one's intentions (Abele \& Wojciszke, 2013) and warmth is the intention toward the perceiver (Fiske, Cuddy, \& Glick, 2007). Together, these dimensions tell the perceiver whether the target wishes well or harm (warmth) and whether he or she is capable of following through on those wishes (competence). Social evaluations of competence and warmth of a social group account for the majority of variance in trait attributions to group members (Fiske et al., 2002; Wojciszke \& Abele, 2008; Wojciszke, Bazinska, \& Jaworski, 1998), affecting stereotypes, prejudice (Cuddy, Fiske, \& Glick, 2007; Fiske et al., 2002), and intentions toward social groups (Cuddy, Fiske, \& Glick, 2007, 2008).

The link between warmth and competence and the basic goals of social perception implies a level of primacy for these characteristics (Abele \& Wojciske, 2014). Not only are these 
traits central to social perceptions, but they also have behavioral consequences. When perceived in light of their stereotyped social group membership, social groups' perceived warmth elicits active behaviors, such as protecting or attacking (Cuddy, Fiske, \& Glick, 2007), whereas their perceived competence elicits more subtle, passive behaviors, such as cooperation with competent groups or exclusion of incompetent groups (Cuddy et al., 2007). The Behaviors from Intergroup Affect and Stereotypes (BIAS) model (Cuddy et al., 2007) shows that the basic dimensions of social perception differentially predict two dimensions of behavior: valence and intensity. Intensity captures how overt and effortful actions are and ranges from active to passive (Cuddy et al., 2007). Perceived warmth predicts active behaviors, because warmth information is more immediately relevant, possibly requiring urgent action. Conversely, competence information is less self-relevant and therefore is less likely to require immediate action, so its perception predicts passive behaviors (Fig. 1, left panel; Cuddy et al., 2007). Research on these behaviors has successfully predicted passive and active intentions toward stereotyped groups based on the groups' perceived competence and warmth (Cuddy et al., 2007; Kervyn, Fiske, \& Yzerbyt, 2015; Fiske, 2015). This research focused on behaviors toward social groups instead of individuals, so the present work tests whether similar relationships hold for intentions toward individuals who vary on the dimensions of warmth and competence.

Interestingly, perceptions of competence and warmth also affect neural processing. For instance, the perceived misfortune of members of a social outgroup that is perceived as high in competence and low in warmth predicts increased activity in areas of the brain associated with subjective pleasure (Cikara \&
Fiske, 2012), while those outgroup members perceived as low in both warmth and competence elicit deactivation of brain areas involved in social cognition (Harris \& Fiske, 2006, 2007, 2011). Together these findings suggest a compelling idea: our basic perceptions of the warmth and competence of a target's social group determines how we think about and treat the individual. An open question, then, is how warmth and competence might affect the social perception of and interaction with individuals independent of group membership. Hence, the objective of the current study was to manipulate warmth and competence perceptions of individual targets directly to investigate the effects on 1) action-related neural processing and 2) intentions toward those specific individuals.

\section{Neural motor resonance}

An important neural process involved in social interaction is motor resonance, the vicarious activation of an observer's sensorimotor and pre-motor areas during observation of another's actions (Avenanti, Candidi, \& Urgesi, 2013; Sartori, Begliomini, Panozzo, Garolla, \& Castiello, 2014; Uithol, van Rooij, Bekkering, \& Haselager, 2011). Motor resonance is thought to contribute to many important social functions, including imitation (Iacoboni, 2005), joint action (Sebanz, Bekkering, \& Knoblich, 2006), and empathy (Moore \& Franz, 2017; Pineda \& Hecht, 2009). Motor resonance may be elicited by others' pain (Bufalari, Aprile, Avenanti, Di Russo, \& Aglioti, 2007; Li, Meng, Li, Yang, \& Yuan, 2017; Perry, Bentin, Bartal, Lamm, \& Decety, 2010) or actions (Aglioti, Cesari, Romani, \& Urgesi, 2008; Bouquet, Shipley, Capa, \& Marshall, 2011; Bowman et al., 2017; Uithol et al.,

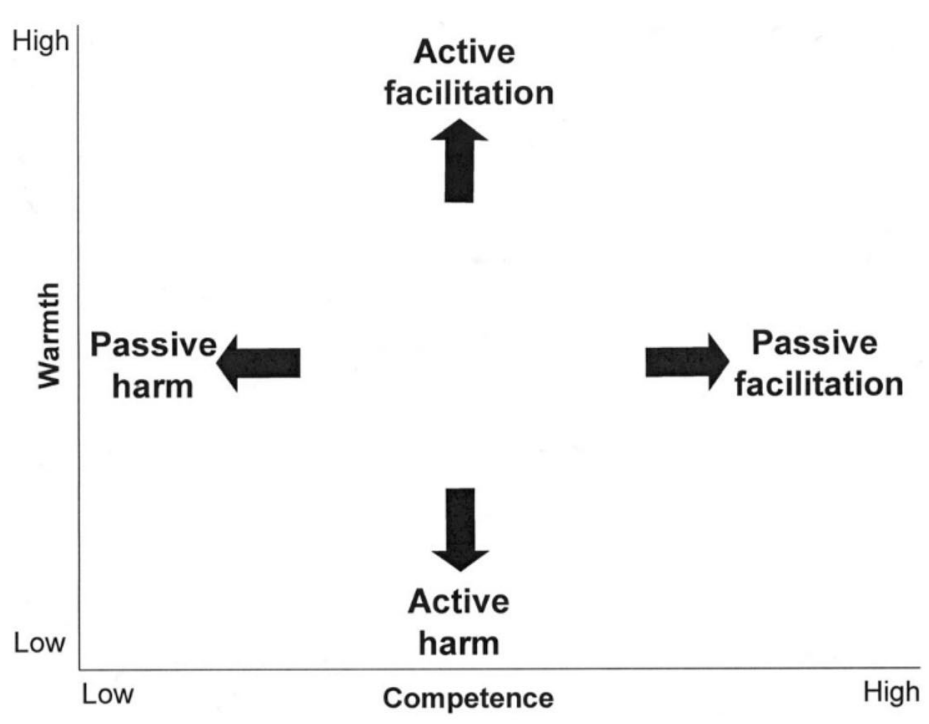

Fig. 1. Left panel: the BIAS map model (adapted from Cuddy et al., 2007); Right panel: participants' actual active and passive intention data (helping minus harm). $\mathrm{C}=$ Competence; $\mathrm{W}=$ Warmth; $\mathrm{H}=$ High; $\mathrm{M}=$

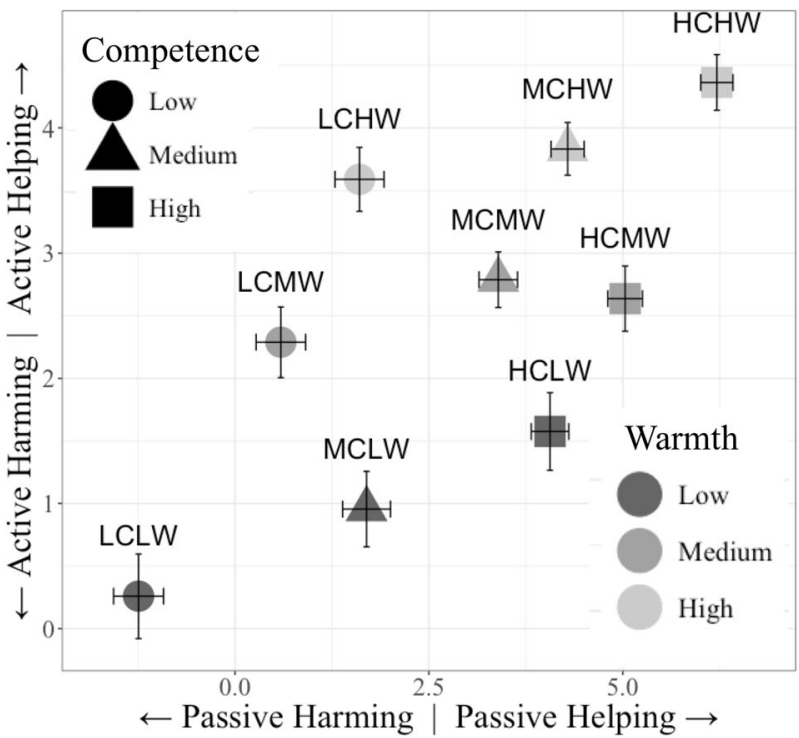

Medium; L = Low. Color indicates degree of warmth; shape indicates degree of competence. Error bars indicate standard error of the mean differences 
2011) and is considered an index of neural mirroring, an important process by which we encode others' actions and intentions (Bowman et al., 2017; Fox et al., 2015). Most relevant to the current research, motor resonance is responsive to the social interaction context (Hogeveen \& Obhi, 2011, 2012; Perry, Stein, \& Bentin, 2011) and predicts participants' movements in response to others (Aridan, Ossmy, Buaron, Reznik, \& Mukamel, 2018), as well as subsequent coordinated action (Naeem, Prasad, Watson, \& Kelso, 2011) and cooperation (Endedijk, Meyer, Bekkering, Cillessen, \& Hunnius, 2017), suggesting that motor resonance is at least associated with more complex social behavior.

Crucially, motor resonance is affected by top-down social processing and is biased against certain social targets. For instance, people tend to resonate more with racial in-group members (Avenanti, Sirigu, \& Aglioti, 2010; Gutsell \& Inzlicht, 2010, 2013), goal-relevant actions (Cattaneo, Caruana, Jezzini, \& Rizzolatti, 2009), those with a positive social valence (Hommel, Colzato, \& Van Den Wildenberg, 2009), and those with whom they share a common goal (Ménoret et al., 2014). Pertinently, participants also resonate preferentially with those who treat them fairly and with whom they want to affiliate (Aragón, Sharer, Bargh, \& Pineda, 2013). This evidence suggests that both high competence and high warmth targets should elicit more resonance.

On the other hand, angry movements elicit more neural motor processing than neutral movements (Engelen, Zhan, Sack, \& Gelder, 2018) and recognition of negative emotions slows down when motor processing is disrupted though transcranial magnetic stimulation (TMS; Balconi \& Bortolotti, 2013). Therefore, motor resonance might not be limited to prosocial contexts but rather be driven by the motivational relevance of a target (Gutsell \& Inzlicht, 2013). Such a functional perspective on person perception and motor resonance asserts that people allocate their limited cognitive resources to social targets who are relevant to their own goals and who might provide functionally significant information (Ackerman et al., 2006; Ohman, Flykt, \& Esteves, 2001; Varnum, Blais, \& Brewer, 2016). Accordingly, we expected low warmth to elicit greater motor resonance because illintentioned, threatening targets are more motivationally relevant.

We had divergent hypotheses about the relationship between motor resonance and high warmth because theory and empirical findings suggest different outcomes; a functional theory of motor resonance would predict that prosocial intentions of a warm target may elicit motor resonance because their actions might benefit the observer. However, preliminary findings from our laboratory show that, although threatening actions elicited significantly stronger motor resonance than neutral actions, positive action did not elicit increased motor resonance (Gutsell \& Inzlicht, 2013). Moreover, others have found that disrupting motor resonance leads to longer reaction times in an emotion recognition task for fearful and angry facial expressions, whereas happy expressions remained unaffected (Balconi \& Bortolotti, 2013). Hence, we predicted either a quadratic trend for warmth with both high and low warmth targets eliciting increased motor resonance compared to medium warmth targets or a linear trend with only low warmth targets eliciting motor resonance.

A common resonance measure uses electroencephalography (EEG) to quantify motor resonance as a decrease in power within the mu frequency band $(8-13 \mathrm{~Hz})$ at electrodes positioned over the sensorimotor cortex (Fox et al., 2015). Mu waves originate from brain areas that activate during actionexecution and action-perception (Cochin, Barthelemy, Roux, \& Martineau, 1999; Fox et al., 2015; Thorpe, Cannon, \& Fox, 2016), primarily clustered around the central sulcus in sensorimotor and parietal areas (Salmelin \& Hari, 1994). Musuppression in the sensorimotor cortex is considered to be a valid measure of motor resonance (Fox et al., 2015, and Hétu et al., 2013, for meta-analyses) and is the measure of choice for the current study.

We look at motor resonance as a necessarily interactive process, indexing something beyond basic attention or social processing. Because resonance requires the mapping of another person's behavior and/or intentions onto the perceiver's brain, it requires both attention-directed perception and recognition of the other as sufficiently similar to the self to simulate. Investigating the individual and combined effects of competence and warmth on motor resonance can therefore shed light on the relative importance of prosocial versus instrumental motivations in basic processing of actions.

\section{Current research}

We examined how social traits affect neural motor resonance and intentions. We measured mu power while participants viewed videos of targets described as high, neutral, or low in warmth and competence. In critical reviews, Hobson and Bishop $(2016,2017)$ concluded that some studies of musuppression are unable to rule out the possibility that the "mu" signal collected in sensorimotor areas originates as posterior alpha. We controlled for this issue by comparing central electrodes over sensorimotor areas to more frontal and posterior electrodes. The same critical analysis and others (Bowman et al., 2017) specify the need for an appropriate baseline of mu activity to compare to experimental conditions. By manipulating our independent variables within subject, we are able to compare experimental trials to one another, allowing us to look at the relative suppression effect within similar trials rather than suppression compared with baseline. Moreover, the mu-suppression literature has been criticized for too many researcher degrees of freedom in regard to 
analysis parameters and too small sample sizes. To address these issues, the current research is preregistered and adequately powered.

To test intentions in an interpersonal context, we used Asbrock and Cuddy's (unpublished) paradigm (Cuddy, Fiske, \& Glick, 2008) ${ }^{1}$ for measuring active and passive intentions toward individuals rather than groups. While warmth and competence reliably predict intergroup social evaluations, no work has linked them to passive/active intentions toward individuals instead of social groups. Testing intentions to perform these behaviors interpersonally and tying them to neural motor resonance are novel contributions of this work that allow us to measure the effects of competence versus warmth on basic neural processing and preparation of actions, plus potential downstream intentions.

\section{Hypotheses}

Our hypotheses are preregistered at https://osf.io/uka6c. In taking a functional approach to person perception and motor resonance, we expected higher competence social targets to elicit greater motor resonance due to the increased motivational relevance of a capable target. Similarly, we expected low warmth to elicit greater motor resonance because ill-intentioned, threatening targets are more motivationally relevant. Due to our divergent hypotheses about the relationship between motor resonance and high warmth, we preregistered two alternative hypotheses for warmth: a linear main effect of warmth and a quadratic main effect of warmth.

In addition, we anticipated that the intentions measure would separate targets' warmth and competence as predictors of active and passive intentions, respectively; we predicted higher warmth to elicit more helping and fewer harming active intentions, and higher competence to elicit more helping and fewer harming passive intentions. Because the BIAS model describes warmth and competence as independent, non-interacting dimensions (Fiske et al., 2002), we did not predict an interaction between warmth and competence.

Finally, we expected to find a link between motor resonance and intentions such that more resonance would predict more helping and fewer harming intentions. This hypothesis was motivated by a potential link between motor resonance and empathy (Hoenen, Schain, \& Pause, 2013; Perry et al., 2010, Pineda \& Hecht, 2009; Woodruff, Martin, \& Bilyk, 2011) such that participants would be more likely to help those with whom they resonated.

\footnotetext{
${ }^{1}$ The paradigm was published in this review paper, but the results of the study have not been.
}

\section{Methods}

\section{Participants}

The sample size was chosen (and preregistered) in accordance with the Fox et al. (2015) meta-analytic recommendation that a sample of 66 is needed to achieve a moderate effect size $(d=$ 0.31 ) in mu desynchronization during action observation. The same sample size also provides sufficient power for measuring the effect of competence and warmth on intentions, given the larger effect size of $d=0.43$ from Asbrock \& Cuddy's unpublished findings. A power analysis revealed that 58 participants are needed to adequately power our planned repeated measures ANOVA on mu-suppression as a function of warmth and competence. To our knowledge, this is one of the first studies to meet Fox et al.'s recommendation, making it one of the largest mu-suppression studies to date (Hobson \& Bishop, 2016). Sensitivity analysis also shows that our sample is sufficient to find a correlation between intentions and musuppression of $r=0.33$ at 0.80 power.

Sixty-eight members of the university community participated in the study for course credit or financial compensation. Two participants were excluded due to unusable EEG data. ${ }^{2}$ The final sample, therefore, was 66 participants (18 males, 47 females, 1 other; 31 White, 9 East-Asian, 6 Hispanic, 5 Black, 3 South-Asian, 1 Middle-Eastern, 1 other, 10 multiracial; mean age $=18.95$ years, $S D=1.39$; 65 right-handed $^{3}$ ). The university's Institutional Review Board approved the study.

\section{Procedure}

After giving written consent, participants were video recorded while repeatedly squeezing a stress ball. They were told they would be learning about previous participants and watching similar videos of them. Participants were then asked to choose which adjectives they tend to be perceived as from lists of seven warmth words (friendly. kind, reliable, well-intentioned, enthusiastic, sincere, and tolerant) and seven competence words (decisive, capable, independent, persuasive, skillful, confident, and efficient; adapted from Horchak, Giger, \& Garrido, 2016) as a personality test. This served to define competence and warmth for the participants, reinforced the cover story that the target individuals were other participants, and provided the rationale for the warmth and competence scores (low, medium, and high) of nine target individuals, each identified with a letter

\footnotetext{
${ }^{2}$ Preregistered outlier analysis identified four participants who were over two standard deviations from the mean in mu power at baseline. Removing them had minor effects on main analyses and we lacked explanations for their extreme values, so results are reported with the full sample and differences when outliers are removed are noted.

${ }^{3}$ Removing the one left-handed participant did not affect analyses, so the data were included.
} 
between $\mathrm{A}$ and I. Letters and trait pairings were randomly matched in four sets, which varied between participants. Participants performed a passive viewing (motor resonance) task, in which each target identity was linked with a video of ostensibly the target's hand squeezing a stress ball, so that variations in the target's warmth and competence scores might affect motor resonance in response to the paired action videos. In this task, each identity was introduced as a silhouette with a warmth score (low, medium, or high) and a competence score (low, medium, or high), followed by the ball-squeezing video (Fig. 2). We chose this less ecological approach rather than descriptions of warm versus competent traits to control participants' perceptions of the targets as directly as possible and to have three levels of each dimension to allow us to find quadratic effects. Participants then completed an intentions task asking them to report the likelihood that they would help or harm each of the target individuals from the motor resonance task in a hypothetical classroom scenario (Asbrock \& Cuddy, unpublished; Cuddy et al., 2008). Finally, participants completed additional exploratory questionnaires which are listed in the supplementary materials. Participants wore the EEG cap throughout the study. See the Open Science Framework (https://osf.io/kucjd/) for the text of all of our measures.

\section{Motor resonance task}

In the motor resonance task, trials consisted of jittered whitenoise for 2,000-2,300 ms, followed by a fixation cross for 500 $\mathrm{ms}$, and 4,000 ms of target information: a letter identifier, a silhouette (the same for all targets) and warmth (low, medium, or high) and competence (low, medium, or high) scores resulting in nine conditions of warmth and competence combinations. This was followed by a 500-ms fixation cross and a video of a hand squeezing a stress ball for 2,000 ms (Fig. 2). Each of the nine target video combinations was presented 14 times for a total of 126 trials broken into seven blocks with short, participant-controlled breaks between each block. The action videos depicted a right hand squeezing a stress ball at a rate of approximately one squeeze per second. All hands were white, evenly split between genders, and depicted from the wrist down with no identifying markers. Within each randomization set, each identity was always shown with the same video. The hands' gender was counterbalanced between conditions so that "Participant A" was female exactly half of the time.

\section{Measures}

\section{Intentions measure}

To assess how mu-suppression might predict intentions previously linked with warmth and competence, we measured participants' willingness to actively/passively help/harm the target individuals (adapted from Cuddy and Asbrock, unpublished). For each target identity, participants were asked to imagine taking a course in which $60 \%$ of their final grade would be determined by a joint project with another student. They were asked to imagine being assigned to work with each target and to assess the likelihood that they would behave in various ways toward that individual on a five-point scale from "not at all likely" to "very likely." They read that "the person assigned to work with you is Participant A [B-I], who is clearly one of the smartest [least smart; of average intelligence] and most competent [least competent; of average competence] students in the class, and also is friendly [unfriendly; neither friendly nor unfriendly] and warm [cold; neither warm not cold] toward the other students." Each identity's description matched the version presented in the motor resonance task and participants answered the same questions about all nine identities. Their choices fell into four categories, with two actions in each category: active help ("share notes with partner," "do more work to help my partner"), active harm ("take undue credit for my partner's work," "tell my professor (i.e. tattle) if I don't think my

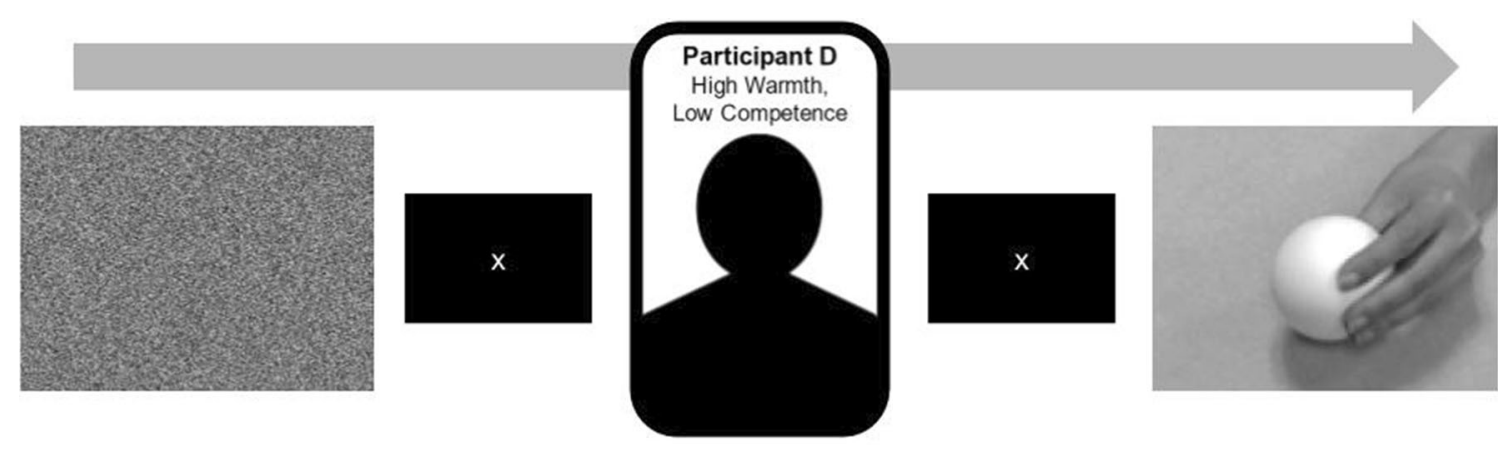

2000-2300ms $500 \mathrm{~ms}$ $4000 \mathrm{~ms}$ $500 \mathrm{~ms}$ $2000 \mathrm{~ms}$

Fig. 2. Trial sequence. Participants viewed a sequence of white noise (jittered between 2,000 and 2,300 ms), fixation cross, silhouette with warmth and competence information, fixation cross, and motor action video for each trial 
partner is doing his or her share"), passive help ("accept my partner's ideas, " "take my partner's ideas seriously"), and passive harm ("avoid meeting with my partner," "dismiss my partner's ideas"). Responses for both actions in each category were added, yielding four scores per participant, bounded at two and ten. Principal component analysis with varimax rotation for each target identity confirmed that the eight items fit well into two factors corresponding to passive and active intentions with average eigenvalues of 2.80 and 1.77. Helping intentions loaded positively and harming intentions loaded negatively on each factor, except for the first active harm item which loaded poorly on both factors.

\section{EEG recording and processing}

We recorded EEG from 33 active electrodes embedded in a stretch-lycra cap (ActiCap, BrainProducts GmbH, Munich, Germany), arranged according to the 10-20 system with impedances below $10 \mathrm{~K} \Omega$. The EEG was digitized at $500 \mathrm{~Hz}$ using BrainAmp amplifiers and BrainVision Recorder software (BrainProducts GmbH, Munich, Germany) with an initial reference at $\mathrm{FCz}$, then rereferenced offline to an average reference as part of processing and analysis in BrainVision Analyzer (BrainProducts GmbH, Munich, Germany). Vertical electrooculogram (VEOG) was recorded with a pair of electrodes placed above and below the right eye. EEG recordings were filtered with a 0.01 to $30 \mathrm{~Hz}(24 \mathrm{~dB} /$ oct $)$ bandpass filter, large artifacts were automatically identified and excluded, and data was corrected offline for eye-blinks using the VEOG channels and an independent component analysis-based procedure for isolating and removing ocular artifacts (Croft \& Barry, 2000). Remaining artifacts were automatically rejected when data included voltage steps greater than $200 \mu \mathrm{V} / \mathrm{ms}$, absolute amplitudes greater than $160 \mu \mathrm{V}$, and activity levels lower than $0.5 \mu \mathrm{V} / 100 \mathrm{~ms}$. Artifact-free epochs of $250 \mathrm{~ms}$ were extracted from each two-second stimulus presentation (taken from 0-2000ms post-stimulus onset) through a $25 \%$ Hamming window and overlapped by $75 \%$ to minimize data loss. We performed a fast Fourier transformation and calculated power in the $8-13 \mathrm{~Hz}$ band for each video presentation. ${ }^{4}$ We then averaged spectral data for videos within each of the nine competence/warmth targets and across the white-noise baseline. Trials with artifacts were not included, resulting in $<1.5 \%$ data loss. Power values were log transformed to normalize the data.

\footnotetext{
${ }^{4}$ A typo in our preregistration listed $8-16 \mathrm{~Hz}$. This was a mistake, as mu is commonly agreed to be $8-13 \mathrm{~Hz}$ (Fox et al., 2015).
}

\section{Results}

\section{Intentions measure}

Our preregistered hypotheses were that warmth and competence would affect active versus passive helping and harming intentions differently, with increasing competence facilitating passive helping and reducing passive harming and increasing warmth facilitating active helping and reducing active harming. To simplify this analysis, we created a preregistered index of positive intentions by subtracting harming intentions from helping intentions for each target identity (see the Supplemental Materials for the analysis without difference scores). We then ran a 3 (target competence: low, medium, high) $x 3$ (target warmth: low, medium, high) x 2 (intention type: active or passive) repeated measures factorial ANOVA. Our preregistered predictions were an interaction between warmth and type, with higher warmth predicting increased active positive intentions, and an interaction between competence and type, with higher competence predicting increased passive positive intentions. We did not predict interactions between warmth and competence because the BIAS model describes the traits as independent from each other. Means and standard deviations for each intention are in Table 1 (and Table S1 breaks them down by target).

The ANOVA revealed main effects of competence, $F(1.47$, $95.48)=99.16, p<0.001, \eta_{p}{ }^{2}=0.60$, and warmth, $F(1.46$, $95.16)=124.00, p<0.001, \eta_{p}{ }^{2}=0.66 .^{5}$ These were qualified by interactions between competence and type, $F(1.57,101.73)$ $=90.09, p<0.001, \eta_{p}{ }^{2}=0.58$, and competence and warmth, $F(4,260)=3.34, p=0.011, \eta_{p}{ }^{2}=0.049$. The predicted interaction between warmth and type did not emerge, ${ }^{6} F(1.70$, $110.72)=2.09, p=0.14, \eta_{p}{ }^{2}=0.031$, nor was there a threeway interaction, $F(3.58,232.48)=0.23, p=0.90, \eta_{p}{ }^{2}=0.004$. These results showed that, as predicted, competence more strongly affected passive than active intentions (simple effect of competence on passive intentions: $F(2,64)=80.70, p<$ $0.001, \eta_{p}{ }^{2}=0.72$; simple effect of competence on active intentions: $F(2,64)=6.45, p=0.003, \eta_{p}{ }^{2}=0.17$; Fig. 3$)$, but that, contrary to predictions, warmth similarly affected both active and passive intentions (simple effect of warmth on passive intentions: $F(2,64)=72.97, p<0.001, \eta_{p}{ }^{2}=0.70$; simple effect of warmth on active intentions: $F(2,64)=52.47, p<$ $0.001, \eta_{p}{ }^{2}=0.62$ ). The unexpected interaction of competence and warmth was driven by targets that were high competence, medium warmth being relatively more similar to medium competence, medium warmth targets, though planned comparisons showed that all identities differed significantly, all $p \mathrm{~s}<0.01$.

\footnotetext{
${ }^{5}$ Except for the competence/warmth interaction, $p$-values reported here are Greenhouse-Geisser corrected, as the assumption of sphericity was violated. ${ }^{6}$ This interaction is marginal when excluding outliers.
} 
Table 1. Means and standard deviations for all four intentions

\begin{tabular}{ll}
\hline Intention & Mean (SD) \\
\hline Active Harm & $4.91(1.48)$ \\
Active Help & $7.39(1.90)$ \\
Passive Harm & $4.66(1.88)$ \\
Passive Help & $7.51(1.99)$ \\
\hline
\end{tabular}

\section{Motor resonance}

We found significant suppression within the mu-frequency band during action observation $\left(M=0.69 \mathrm{mV}^{2}, S D=0.29\right.$ $\left.\mathrm{mV}^{2}\right)$ compared with baseline $\left(M=0.92 \mathrm{mV}^{2}, S D=0.32\right.$ $\mathrm{mV}^{2}$ ) within our region of interest, the left central electrode $\mathrm{C} 3, t(65)=-17.65, p<0.001, d=2.17$, which is above the sensorimotor cortex contralateral to the right hands participants watched. The odd-even split half reliability of musuppression (calculated as log ratio of observation/baseline) was .96 , indicating that we were able to obtain a reliable measure of mu-suppression. To test the localization of this effect and its response to the dimensions of social perception, we compared suppression across frontal (Fz, F3, F4), central (Cz, $\mathrm{C} 3, \mathrm{C} 4)$, and parietal (Pz, P3, P4) electrodes, performing a 3 (Warmth: high, medium, low) x 3 (Competence: high, medium, low) x 3 (Electrode centrality: Frontal, Central, Parietal) x 3 (Electrode lateralization: left, central, right) repeatedmeasures ANCOVA on mu-power, covarying for baseline white-noise across electrodes. ${ }^{7}$ Results revealed a significant main effect of centrality, $F(2,128)=16.62, p<0.001, \eta^{2}=$ 0.21 , and a marginal interaction of warmth and competence, $F(4,256)=2.29, p=0.06, \eta^{2}=0.035 .{ }^{8}$ Preregistered simple comparisons showed more mu-suppression in central electrodes than frontal $(p<0.001)$ or parietal $(p<0.001)$, and significant differences between left, central, and right electrodes (all $p \mathrm{~s}<0.01$ ), with the most mu-suppression at left electrodes. Within the central electrodes, there was significantly more mu-suppression at $\mathrm{C} 3\left(M=0.69 \mathrm{mV}^{2}, S E=0.017\right)$ and $\mathrm{C} 4(M=0.74, S E=0.017)$ than at $\mathrm{Cz}(M=0.84, S E=$ 0.014; $p \mathrm{~s}<0.001)$, though the difference between $\mathrm{C} 3$ and $\mathrm{C} 4$ was not significant $(p=0.43)$, suggesting that our effects did not lateralize as much as expected.

\footnotetext{
${ }^{7}$ This analysis was preregistered. A post-hoc comparison of central to occipital electrodes in line with the recommendations of Hobson and Bishop (2016) in a similar ANCOVA found a main effect of electrode centrality, $F(1,62)=$ $4.56, p=0.037, \eta^{2}=0.069$, showing greater suppression of the $8-13 \mathrm{~Hz}$ wave in central than occipital regions. This suggests that the effect on motor resonance is separate from possible attentional differences indexed by occipital alpha. There was also a main effect of laterality, $F(2,124)=5.34, p=0.006$, $\eta^{2}=0.079$, and an interaction between centrality and laterality, $F(2,124)=$ 7.63, $p=0.001, \eta^{2}=0.11$, with greater suppression on the sides than in the middle, especially at central electrodes.

${ }^{8}$ Excluding outliers removed the marginal warmth/competence interaction and provided a marginal effect of lateralization $(p=0.082)$. The simple effects do not change, nor does the second ANCOVA below.
}

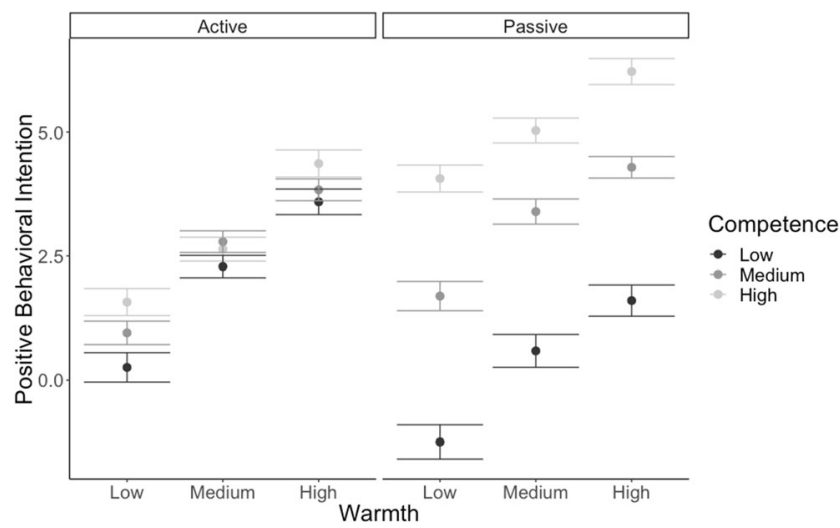

Fig. 3. Positive intentions (help - harm) for each level of warmth and competence, split between active and passive intentions. Error bars indicate standard error of the mean.

With this evidence of resonance, we then ran a 3 (Warmth: high, medium, low) x 3 (Competence: high, medium, low) ANCOVA with the $\mathrm{C} 3$ white-noise baseline as the covariate, looking at mu-suppression at $\mathrm{C} 3$. This analysis revealed no significant main effects nor interaction ( $p$ s >0.33). Nonetheless, we proceeded with our a priori preregistered planned comparisons (Tybout et al., 2001; see "Follow-up analyses" in the preregistration). For high competence targets, there was a quadratic effect of warmth with participants showing significantly more mu-suppression for low than medium warmth targets, $p=0.044, d=0.26$, and significantly more mu-suppression for high than medium warmth targets, $p=$ $0.031, d=0.26$. Similarly, for medium warmth targets, there was a linear effect of competence with participants showing significantly more mu-suppression for high competence targets than for low competence, $p=0.019, d=0.29$, or medium competence targets, $p=0.003, d=0.38$. This is a more nuanced result than our predicted main effects of competence and of warmth, but the pattern we expected did appear: high competence generated the most resonance when targets were perceived as neither very warm nor very cold, and warm and cold targets elicited more resonance than neutral targets when they were perceived as highly competent (Fig. 4).

Finally, we looked for a potential relationship between intentions and mu-suppression. Neither active nor passive positive intentions correlated with mu-suppression (active: $r(64)$ $=0.16, p=0.20$; passive: $r(64)=-0.15, p=0.20)$. Thus, we failed to detect a relationship between intentions and musuppression.

\section{Discussion}

Warmth and competence have been identified as primary dimensions of social perception, and their effects on perception and social cognition have been investigated for decades (Judd et al., 2005), but the underlying neural processes and 


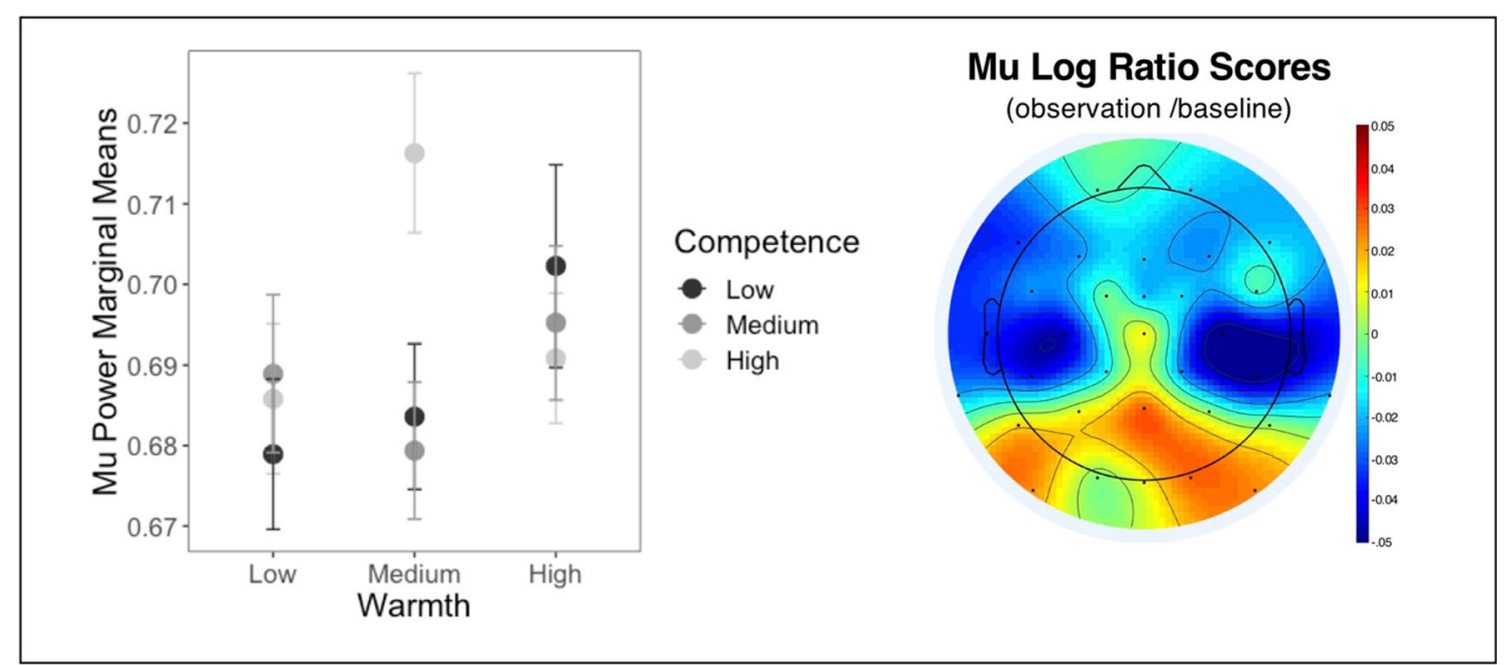

Fig. 4. Left panel: Mu power during action observation trials for each target identity. Values are marginal means controlling for average baseline activity, generated from the reported ANCOVA. High competence targets elicited less mu, and thus more resonance, when they were high or low warmth compared with medium warmth. Similarly, medium warmth

downstream effects on intentions and behavior, particularly in an interpersonal context, are still poorly understood. We have provided evidence of separate and combined effects of warmth and competence on intentions and neural resonance. Targets' perceived warmth and competence predicted active and passive intentions toward them, replicating previous, unpublished findings (Cuddy et al., 2008), and predicted motor resonance at least for medium warmth and high competence targets. To our knowledge, these findings provide the first evidence of personality trait impressions affecting motor resonance.

\section{Effects on motor resonance}

Although the patterns are more nuanced than predicted, our resonance results present the first evidence that neural action processing shows differential responses to perceived warmth and competence. Participants resonated less with high competence, medium warmth social targets than with the other medium warmth or high competence targets. The predicted quadratic effect of warmth was present, but only for high competence targets, suggesting that target warmth matters only when the target is perceived as high in competence. This aligns with a functional account of motor resonance: resonance responds on the basis of targets' social relevance, as determined by their combination of intention and capacity (Ackerman et al., 2006; Fiske, 2018; Ohman et al., 2001). Warmth-based evaluations are considered stable while competence-based evaluations depend on warmth (Abele \& Wojciszke, 2013). Our results suggest that resonance responds to the interaction of warmth and competence, but while perception of warmth drives our attitudes toward others (Abele \& Wojciszke, 2007; Cuddy et al., targets elicited less mu when they were low or medium competence compared to high competence. Error bars indicate standard error of the mean. Right panel: topographic depiction of overall mu-suppression (log ratio scores (observation/baseline) across all target identities)

2008), perceived competence seems to dictate whether warmth affects motor resonance. The responsiveness of motor resonance to dimensions of social perception adds to the growing literature that top-down representations of targets affect the basic processing of their actions (Aragón et al., 2013; Gutsell \& Inzlicht, 2010; Hogeveen, Chartrand, \& Obhi, 2015).

\section{Effects on intentions}

Consistent with the BIAS model (Cuddy et al., 2007), competence affected passive intentions and warmth affected active intentions, even in an interpersonal context. This finding suggests a successful manipulation of warmth and competence on an individual level and indicates a successful replication of warmth and competence predicting intentions (Cuddy et al., 2007, 2008). However, while competence predicted passive more than active intentions, in keeping with the model, our results did not show a stronger association of warmth with active over passive intentions. While we did not predict an interaction between warmth and competence, previous research on warmth and competence indicates that an increase on one dimension predicts a decrease on the other, such that those who are perceived as warmer are less likely to be perceived as competent, but those who are perceived as more competent are less likely to be perceived as warm (Cuddy et al., 2009). Additional studies of warmth and competence do show an interaction between warmth and competence (Durante et al., 2017; Durante et al., 2013; Imhoff \& Koch, 2017), potentially explaining the interaction between warmth and competence in our intentions findings. In sum, our data confirm that perceptions of individuals' warmth and competence can affect intentions and show that warmth is an 
important determinant of both passive and active intentions despite their orthogonality in the BIAS map.

We did not detect a relationship between mu-suppression and intentions. According to the empathetic account of resonance, resonance should increase helping; however, we did not find the expected equivalent positive relationship with more positive intentions. It is possible that by measuring intentions rather than actual behavior we minimized the effect of implicit processes on our intentions measure. Additionally, it is possible that motor resonance does not predict hypothetical interactions or intentions to behave and only relates to direct interaction. Finally, given that motor resonance is tightly linked to sensorimotor processing, it is also possible that it would only predict intentions of concrete motor actions and not more abstract intentions like accepting a partner's ideas. Thus, future studies could benefit from a more direct approach that measures actual behaviors and more basic motor actions rather than intentions about complex social behavior.

\section{Limitations and conclusions}

The manipulation of perceived warmth and competence was designed to target the concepts in their purest forms. This benefit for internal consistency likely comes with decreased external validity. Being labeled as "high warmth" is not the same as actually displaying warm behavior, and being labeled as "low competence" will likely affect social perceptions differently than actually displaying incompetent behavior; perhaps in the real world these dimensions might yield even stronger results due to the greater motivational relevance of the target's traits. In addition, there is some possibility that the ratings of warmth and competence and the reported intentions were both indexing how positively or negatively the targets were perceived, which we did not measure. However, the differentiation of intentions by warmth and competence (Fig. 3 ) leaves us confident that the manipulation and measure were not confounded in this way.

Social perception is inherently connected to interaction. The current findings support this notion by showing that manipulations of the primary dimensions of social evaluation can affect not only how the brain processes a target's actions but also intentions toward the target. Finding that motor resonance - a neural index of action processing linked to action understanding and basic empathy-is affected by the primary dimensions of social perception advances our understanding of social processing. Our results suggest that people's impressions of others' warmth and competence can affect how they relate to others' experiences even on such a basic level as action processing. Moreover, by looking at how warmth and competence affect both motor resonance and intentions, we provide insight into how the top-down and bottom-up processes of social impression formation and action processing might interact to drive decisions about behavior.
Open practices statement The data, materials, and preregistration of this study are available at https://osf.io/kucjd/.

\section{References}

Abele, A. E., \& Bruckmüller, S. (2011). The Bigger one of the "Big Two"? Preferential processing of communal information. Journal of Experimental Social Psychology, 47, 935-948. https://doi.org/ 10.1016/j.jesp.2011.03.028

Abele, A. E., \& Wojciszke, B. (2007). Agency and Communion from the Perspective of Self Versus Others. Journal of Personality and Social Psychology, 93(5), 751-763. https://doi.org/10.1037/0022-3514.93. 5.751

Abele, A. E., \& Wojciszke, B. (2013). The big two in social judgment and behavior. Social Psychology, 44(2), 61-62. https://doi.org/10.1027/ 1864-9335/a000137

Abele, A. E., \& Wojciszke, B. (2014). The Big Two in Social Judgment and Behavior. Advances in Experimental Social Psychology (1st ed., Vol. 50). Elsevier Inc. https://doi.org/10.1016/B978-0-12800284-1.00004-7

Ackerman, J. M., Shapiro, J. R., Neuberg, S. L., Kenrick, D. T., Becker, D. V., Griskevicius, V., ... Schaller, M. (2006). They All Look the Same to Me (Unless They're Angry): From Out-Group Homogeneity to Out-Group Heterogeneity. Psychological Science, 17(10), 836-840. https://doi.org/10.1111/j.1467-9280.2006.01790. $\mathrm{X}$

Aglioti, S.M., Cesari, P., Romani, M., \& Urgesi, C. (2008). Action anticipation and motor resonance in elite basketball players. Nature Neuroscience, 11(9), 1109-1116. Doi:https://doi.org/10.1038/nn. 2182

Aragón, O. R., Sharer, E. A., Bargh, J. A., \& Pineda, J. A. (2013). Modulations of mirroring activity by desire for social connection and relevance of movement. Social Cognitive and Affective Neuroscience. https://doi.org/10.1093/scan/nst172

Aridan, N., Ossmy, O., Buaron, B., Reznik, D., \& Mukamel, R. (2018). Suppression of EEG mu rhythm during action observation corresponds with subsequent changes in behavior. Brain Research, 5563. https://doi.org/10.1016/j.brainres.2018.04.013

Asch, S. E. (1946). Forming impressions of personality. Journal of Abnormal and Social Psychology, 41, 1230-1240.

Avenanti, A., Candidi, M., \& Urgesi, C. (2013). Vicarious motor activation during action perception: beyond correlational evidence. Frontiers in Human Neuroscience, 7(May), 185. https://doi.org/10. 3389/fnhum.2013.00185

Avenanti, A., Sirigu, A., \& Aglioti, S. M. (2010). Racial bias reduces empathic sensorimotor resonance with other-race pain. Current Biology: $C B, 20(11), 1018-22$. https://doi.org/10.1016/j.cub.2010. 03.071

Balconi M., \& Bortolotti A. (2013) The "simulation" of the facial expression of emotions in case of short and long stimulus duration: The effect of pre-motor cortex inhibition by rTMS. Brain $\operatorname{Cog} n$ 83, 114 120. Doi:https://doi.org/10.1016/j.bandc.2013.07.003

Bouquet, C.A., Shipley, T.F., Capa, R.L., \& Marshall, P.J. (2011). Motor contagion: goal-directed actions are more contagious than non-goaldirected actions. Experimental Psychology, 58(1), 71-78. Doi: https://doi.org/10.1027/1618-3169/a000069

Bowman, L. C., Bakermans-Kranenburg, M. J., Yoo, K. H., Cannon, E. N., Vanderwert, R. E., Ferrari, P. F., ... Fox, N. A. (2017). The murhythm can mirror: Insights from experimental design, and looking past the controversy. Cortex, 96, 121-125. https://doi.org/10.1016/j. cortex.2017.03.025 
Bufalari, I., Aprile, T., Avenanti, A., Di Russo, F., \& Aglioti, S. M. (2007). Empathy for Pain and Touch in the Human Somatosensory Cortex. Cerebral Cortex, 17(11), 2553-2561. https://doi.org/10. 1093/cercor/bhl161

Cattaneo, L., Caruana, F., Jezzini, A., \& Rizzolatti, G. (2009). Representation of Goal and Movements without Overt Motor Behavior in the Human Motor Cortex: A Transcranial Magnetic Stimulation Study. Journal of Neuroscience, 29(36), 11134-11138. https://doi.org/10.1523/JNEUROSCI.2605-09.2009

Cikara, M., \& Fiske, S. T. (2012). Stereotypes and Schadenfreude: Affective and physiological markers of pleasure at outgroup misfortunes. Social Psychological and Personality Science, 3(1). https:// doi.org/10.1177/1948550611409245

Cochin, S., Barthelemy, C., Roux, S., \& Martineau, J. (1999). Observation and execution of movement: similarities demonstrated by quantified electroencephalography. Neuroscience, 11(June 1998), 1839-1842. https://doi.org/10.1046/j.1460-9568. 1999.00598.x

Croft, R. J., \& Barry, R. J. (2000). Removal of ocular artifact from the EEG: a review. Clinical Neurophysiology, 30, 5-19.

Cuddy, A. J. C., Fiske, S. T., \& Glick, P. (2007). The BIAS map: behaviors from intergroup affect and stereotypes. Journal of Personality and Social Psychology, 92(4), 631-648. https://doi.org/10.1037/ 0022-3514.92.4.631

Cuddy, A. J. C., Fiske, S. T., \& Glick, P. (2008). Warmth and Competence as Universal Dimensions of Social Perception: The Stereotype Content Model and the BIAS Map. Advances in Experimental Social Psychology (Vol. 40). https://doi.org/10.1016/S00652601(07)00002-0

Cuddy, A. J. C., Fiske, S. T., Kwan, V. S. Y., Glick, P., Demoulin, S., Leyens J.-P., ... Ziegler, R. (2009). Stereotype content model across cultures: Towards universal similarities and some differences. British Journal of Social Psychology 48 (1):1-33

Endedijk, H. M., Meyer, M., Bekkering, H., Cillessen, A. H. N., \& Hunnius, S. (2017). Neural mirroring and social interaction: Motor system involvement during action observation relates to early peer cooperation. Developmental Cognitive Neuroscience, 24, 33-41. https://doi.org/10.1016/j.den.2017.01.001

Durante, F., Crippa, F., Larsen, C. A., Bye, H. H., Fiske, S. T., Björklund, F., ... Mähönen, T. A. (2017). Ambivalent stereotypes link to peace, conflict, and inequality across 38 nations. Proceedings of the National Academy of Sciences, 114(4), 669-674. https://doi.org/ 10.1073/pnas.1611874114

Durante, F., Fiske, S. T., Kervyn, N., Cuddy, A. J. C., Akande, A. D., Adetoun, B. E., ... Storari, C. C. (2013). Nations' income inequality predicts ambivalence in stereotype content: How societies mind the gap. British Journal of Social Psychology, 52(4), 726-746. https:// doi.org/10.1111/bjso.12005

Engelen T., Zhan M., Sack A. T., \& de Gelder, B. (2018). Dynamic interactions between emotion perception, action perception, and action preparation areas for reacting to social threat: a combined cTBSfMRI study. eNeuro 5:ENEURO.0408-17.2018. https://doi.org/10. 1523/ENEURO.0408-17.2018

Fiske, S. T. (2015). Intergroup biases: a focus on stereotype content. Current Opinion in Behavioral Sciences 3, 45-50.

Fiske, S. T., Cuddy, A. J. C., \& Glick, P. (2007). Universal dimensions of social cognition: warmth and competence. Trends in Cognitive Sciences, 11 (2), 77-83. https://doi.org/10.1016/j.tics.2006.11.005

Fiske, S. T., Cuddy, A. J. C., Glick, P., \& Xu, J. (2002). A model of (often mixed) stereotype content: Competence and warmth respectively follow from perceived status and competition. Journal of Personality and Social Psychology, 82(6), 878-902. https://doi. org/10.1037//0022-3514.82.6.878

Fiske, S.T. (2018). Stereotype Content: Warmth and Competence Endure. Current Directions in Psychological Science, 27 (2), 67-73. https:// doi.org/10.1177/0963721417738825
Fox, N. A, Bakermans-Kranenburg, M. J., Yoo, K. H., Bowman, L. C., Cannon, E. N., Vanderwert, R. E., ... Van Ijzendoorn, M. H. (2015). Assessing Human Mirror Activity With EEG Mu Rhythm: A MetaAnalysis. Psychological Bulletin. https://doi.org/10.1037/ bul0000031

Gutsell, J. N., \& Inzlicht, M. (2010). Empathy constrained: Prejudice predicts reduced mental simulation of actions during observation of outgroups. Journal of Experimental Social Psychology, 46(5), 841-845. https://doi.org/10.1016/j.jesp.2010.03.011

Gutsell, J. N., \& Inzlicht, M. (2013). Using EEG mu-suppression to explore group biases in motor resonance. In B. Derks, D. Scheepers, \& N. Ellemers (Eds.), The Neuroscience of Prejudice (pp. 277-298). London: Psychology Press.

Harris, L. T., \& Fiske, S. T. (2006). Dehumanizing the Lowest of the Low: Neuroimaging Responses to Extreme Out-Groups. Psychological Science, 17(10), 847-853.

Harris, L. T., \& Fiske, S. T. (2007). Social groups that elicit disgust are differentially processed in mPFC. Social Cognitive and Affective Neuroscience, 2(1), 45-51. https://doi.org/10.1093/scan/ns1037

Harris, L. T., \& Fiske, S. T. (2011). Dehumanized perception a psychological means to facilitate atrocities, torture, and genocide? Zeitschrift Fur Psychologie / Journal of Psychology, 219(3), 175181. https://doi.org/10.1027/2151-2604/a000065

Hétu, Sébastien, Gregoire, Mathieu, Saimpont, Arnaud, Coll, MichelPierre, Eugène, Fanny, Michon, Pierre-Emmanuel \& Jackson, Philip. (2013). The neural network of motor imagery: An ALE meta-analysis. Neuroscience and biobehavioral reviews. 37. https://doi. org/10.1016/j.neubiorev.2013.03.017.

Hobson, H. M., \& Bishop, D. V. M. (2016). Mu suppression - A good measure of the human mirror neuron system? Cortex, 1-21. https:// doi.org/10.1016/j.cortex.2016.03.019

Hobson, H. M., \& Bishop, D. V. M. (2017). The interpretation of mu suppression as an index of mirror neuron activity: past, present and future. Royal Society Open Science, 4. https://doi.org/10.1098/rsos. 160662

Hoenen, M., Schain, C., Pause, B. M. (2013). Down-modulation of muactivity through empathic top-down processes. Social Neuroscience, 8(5), 515-524. https://doi.org/10.1080/17470919.2013.833550

Hogeveen, J., \& Obhi, S.S. (2011). Altogether now: Activating interdependent self-construal induces hypermotor resonance. Cognitive Neuroscience, 2(2), 74-82. https://doi.org/10.1080/17588928.2010. 533164

Hogeveen, J., \& Obhi, S.S. (2012). Social Interaction Enhances Motor Resonance for Observed Human Actions. Journal of Neuroscience, 32(17), 5984-5989. https://doi.org/10.1523/JNEUROSCI.5938-11. 2012

Hogeveen, J., Chartrand, T.L., \& Obhi, S.S. (2015). Social Mimicry Enhances Mu-Suppression During Action Observation. Cerebral Cortex. doi:https://doi.org/10.1093/cercor/bhu016

Hommel, B., Colzato, L. S., \& Van Den Wildenberg, W. P. M. (2009). How social are task representations? Psychological Science, 20(7), 794-798. https://doi.org/10.1111/j.1467-9280.2009.02367.x

Horchak, O. V., Giger, J. C., \& Garrido, M. V. (2016). Action contribution to competence judgments: The use of the journey schema. Frontiers in Psychology, 7(MAR). https://doi.org/10.3389/fpsyg.2016.00448

Iacoboni, M. (2005). Neural mechanisms of imitation. Current Opinion in Neurobiology, 15(6), 632-637. https://doi.org/10.1016/j.conb.2005. 10.010

Imhoff, R., \& Koch, A. (2017). How Orthogonal Are the Big Two of Social Perception? On the Curvilinear Relation Between Agency and Communion. Perspectives on Psychological Science, 12(1), 122-137. https://doi.org/10.1177/1745691616657334

Judd, C. M., James-Hawkins, L., \& Yzerbyt, V. (2005). Fundamental Dimensions of Social Judgment : Understanding the Relations Between Judgments of Competence and Warmth. Journal of 
Personality and Social Psychology, 89(6), 899-913. https://doi.org/ 10.1037/0022-3514.89.6.899

Kervyn, N., Fiske, S., \& Yzerbyt, V. (2015). Forecasting the Primary Dimension of Social Perception. Social Psychology 46(1), 36-45.

Knoblich, G., \& Sebanz, N. (2006). The Social Nature of Perception and Action. Current Directions in Psychological Science, 15(3), 99104. https://doi.org/10.1111/j.0963-7214.2006.00415.x

Li, X., Meng, X., Li, H., Yang, J., \& Yuan, J. (2017). The impact of mood on empathy for pain: Evidence from an EEG study. Psychophysiology, 54(9), 1311-1322. https://doi.org/10.1111/psyp. 12882

Ménoret, M., Varnet, L., Fargier, R., Cheylus, A., Curie, A., des Portes, V., ... Paulignan, Y. (2014). Neural correlates of non-verbal social interactions: A dual-EEG study. Neuropsychologia, 55(1), 85-97. https://doi.org/10.1016/j.neuropsychologia.2013.10.001

Moore, M. R., \& Franz, E. A. (2017). Mu rhythm suppression is associated with the classification of emotion in faces. Cognitive, Affective, \& Behavioral Neuroscience, 224-234. https://doi.org/10.3758/ s13415-016-0476-6

Naeem, M., Prasad, G., Watson, D. R., \& Kelso, J. A. S. (2011). Electrophysiological signatures of intentional social coordination in the 10-12 Hz range. NeuroImage. doi:https://doi.org/10.1016/j. neuroimage.2011.08.010

Ohman, A., Flykt, A., \& Esteves, F. (2001). Emotion Drives Attention: Detecting the Snake in the Grass. Journal of Experimental Psychology: General, 130(3), 466-478. https://doi.org/10.1037/ AXJ96-3445.130.3.466

Perry, A., Bentin, S., Bartal, I. B-A., Lamm, C., \& Decety, J. (2010). "Feeling" the pain of those who are different from us: Modulation of EEG in the mu/alpha range. Cognitive, Affective, \& Behavioral Neuroscience, 10(4), 493-504. doi:https://doi.org/10.3758/CABN. 10.4.493

Perry, A., Stein, L., \& Bentin, S. (2011). Motor and attentional mechanisms involved in social interaction-Evidence from mu and alpha EEG suppression. NeuroImage 58(3), 895-904

Pineda, J. A., \& Hecht, E. (2009). Mirroring and mu rhythm involvement in social cognition: Are there dissociable subcomponents of theory of mind? Biological Psychology, 80(3), 306-314. https://doi.org/10. 1016/j.biopsycho.2008.11.003
Salmelin, R., \& Hari, R. (1994). Spatiotemporal characteristics of sensorimotor neuromagnetic rhythms related to thumb movement. Neuroscience, 60(2), 537-550. https://doi.org/10.1016/03064522(94)90263-1

Sartori, L., Begliomini, C., Panozzo, G., Garolla, A., \& Castiello, U. (2014). The left side of motor resonance. Frontiers in Human Neuroscience, 8(September), 1-11. https://doi.org/10.3389/fnhum. 2014.00702

Sebanz, N., Bekkering, H., \& Knoblich, G. (2006). Joint action: Bodies and minds moving together. Trends in Cognitive Sciences, 10(2), 71-76. https://doi.org/10.1016/j.tics.2005.12.009

Thorpe, S. G., Cannon, E. N., \& Fox, N. A. (2016). Rhythms From Infancy Through Adulthood. Journal of Clinical Neurophysiology, 127(1), 254-269. https://doi.org/10.1016/j.clinph.2015.03.004.

Tybout, A. M., Sternthal, B., Keppel, G., Verducci, J., Meyers-Levy, J., \& Barnes, J. (2001). Analysis of Variance. In Iacobucci, D. (Ed.), Journal of Consumer Psychology's Special Issue on Methodological and Statistical Concerns of the Experimental Behavioral Researcher, 10 (1\&2), Mahwah, NJ: Lawrence Eribaum Associates, 5-35.

Uithol, S., van Rooij, I., Bekkering, H., \& Haselager, P. (2011). Understanding motor resonance. Social Neuroscience, 6(4), 388397. https://doi.org/10.1080/17470919.2011.559129

Varnum, M. E. W., Blais, C., \& Brewer, G. A. (2016). Social class affects $\mathrm{Mu}$-suppression during action observation. Social Neuroscience, 11(4), 449-454. https://doi.org/10.1080/17470919.2015.1105865

Wojciszke, B., \& Abele, A. E. (2008). The primacy of communion over agency and its reversals in evaluations. European Journal of Social Psychology Eur., 38, 1139-1147. https://doi.org/10.1002/ejsp

Wojciszke, B., Bazinska, R., \& Jaworski, M. (1998). On the Dominance of Moral Categories in Impression Formation. Personality and Social Psychology Bulletin, 24(12), 1251-1263.

Woodruff, C. C., Martin, T., \& Bilyk, N. (2011). Differences in self- and other-induced $\mathrm{Mu}$ suppression are correlated with empathic abilities. Brain Research, 1405(0), 69-76. https://doi.org/10.1016/j.brainres. 2011.05.046

Publisher's note Springer Nature remains neutral with regard to jurisdictional claims in published maps and institutional affiliations. 